\title{
Changes in plasma oestradiol, testosterone and progesterone concentrations during an annual reproductive cycle in wild Aldabra giant tortoises (Aldabrachelys gigantea)
}

\author{
Maya S. Kummrow ${ }^{1}$, Richard Baxter ${ }^{2}$, Gabriela Mastromonaco ${ }^{3}$, Nancy Bunbury ${ }^{4}$, Marcus \\ Clauss $^{1}$, Dennis Hansen ${ }^{2,5}$ \& Jean-Michel Hatt ${ }^{1}$
}

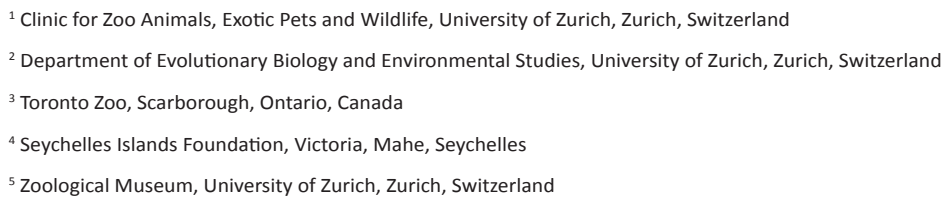

Aldabra giant tortoises (Aldabrachelys gigantea) are currently listed as Vulnerable on the IUCN Red List of Threatened Species. However, negative impacts of sea-level rise are predicted to result in an overall population decline of 40-65\% over the next 100 years, rendering the species Endangered. Captive propagation is an important tool for in- and ex-situ species conservation, but breeding success outside the tortoises' native island habitats has been very limited. Until now, the reproductive cycle of Aldabra tortoises has only been described in anatomic and behavioural studies. During a one-year period, plasma of four female and four male wild tortoises on Aldabra Atoll were examined monthly for levels of gonadal steroid hormones (oestradiol, testosterone, progesterone). Plasma oestradiol and testosterone values as well as meteorological data of the sampling period corresponded to previously published reports on seasonal changes in anatomy, behaviour and climate on the Aldabra Atoll. Seasonal changes in plasma testosterone were evident in males, with high values from January through April, reflecting previously described testicular growth and breeding season, followed by a nadir in August and September. In females, plasma oestradiol levels displayed seasonal changes, coinciding with reported ovarian growth from January to May. The obtained data provide prerequisite knowledge for endocrinological monitoring of reproductive processes and management of breeding programs, both ex-situ as in-situ, to establish reserve- and rewilded populations.

Keywords: Aldabra giant tortoise, Aldabrachelys gigantea, reproduction, seasonality, steroid hormone

\section{INTRODUCTION}

Eary arly human colonisation of the Indian Ocean Islands led to the extinction of one of two lineages of endemic giant tortoises, Cylindraspsis, and the almost complete elimination of the other lineage, Aldabrachelys, by the end of the 19th century due to excessive harvesting and translocation (Gerlach et al., 2013; Hansen et al., 2010). Today, only one species, the Aldabra giant tortoise (Aldabrachelys gigantea), survives. In the 1970s, conservation efforts including population monitoring and research programs were initiated, resulting in a significant increase of the single remaining population on the Aldabra Atoll and introductions of populations outside the historic range (Bourn et al., 1999). Recent introductions of captive-bred $A$. gigantea to new island habitats, such as lle aux Aigrettes and Round Island in Mauritius, have not only served the conservation of the species, but also the restoration of ecosystem function, especially in regard to plant seed dispersal (Falcón \& Hansen, 2018; Falcon et al., 2018; Hansen et al., 2010). However, natural density-related population regulatory mechanisms, late onset of reproductive age, and the extremely restricted range render $A$. gigantea population dynamics vulnerable to external impacts, be they human or natural in origin (Bourn, 1977; Gerlach et al., 2013; Haverkamp et al., 2017). The species is currently listed as Vulnerable on the IUCN Red List of Threatened Species (IUCN, 2018), but an overall population decline of $40-65 \%$ over the next 100 years is projected due to severe negative impacts of sea-level rise, which will render the species Endangered by IUCN criteria (Gerlach et al., 2013). Captive propagation is an important tool for in- and ex-situ species conservation. However, breeding success outside the Aldabra tortoises' native island habitats has been very limited. Of the 159 zoological institutions registered as holding Aldabra tortoises, only one institution (Tulsa Zoo, USA) has been producing and 
distributing offspring to a significant degree in the last 15 years (ZIMS, 2018).

Aldabra tortoises are long-lived species with late onset of sexual maturity at approximately 20 years of age. In their natural habitat on Aldabra Atoll in the southern hemisphere, they show a distinct seasonal reproductive pattern with the mating season in December to March and nesting season from June to September, resulting in prolific numbers of offspring, but with high neonatal mortality, mostly due to predation (Swingland \& Coe, 1978).

Knowledge of the reproductive physiology of Aldabra giant tortoises is based on behavioural observations and morphological examinations of gonads of freshly killed, free-ranging animals on the Aldabra Atoll (Bourn, 1977; Swingland \& Coe, 1978). In captive animals in one zoological institution in the Northern hemisphere, ultrasonographic evaluation of the ovarian cycle and faecal steroid metabolite measurements revealed evidence for asynchrony of the follicular development compared to the seasonal reproductive cycle observed in the native habitat, and follicular atresia (Casares, 1995).

In contrast to the other giant tortoise species, the Galápagos giant tortoise (Chelonoidis niger), of which the reproductive cycle has been studied more intensively both under free-ranging as well as captive conditions (Rostal et al., 1998; Schramm et al., 1999), the endocrinological pattern of the Aldabra giant tortoise has not yet been described.

Endocrinological patterns are a crucial measurement tool for the understanding of reproductive processes, and the present study served to complement the biological observations with endocrinological reproductive patterns in wild Aldabra giant tortoises during an entire year. This is a prerequisite for endocrinological monitoring of reproductive processes, developing hormonal interventions to increase reproductive output, and evaluation of husbandry and management. In particular, knowledge is required on the role of environmental factors, circadian and annual light cycles, and resource availability for the breeding success in captive populations, both ex-situ and in-situ, to establish reserve- and reintroduction populations.

\section{METHODS}

This study was part of a multidisciplinary project on Aldabra giant tortoises on Aldabra Atoll (Falcón et al., 2018; Haverkamp et al., 2017; Turnbull et al., 2015; Walton et al., 2019). The research and sampling protocol were approved by the Seychelles Bureau of Standards.

\section{Animals}

From January to December 2013, eight wild adult Aldabra tortoises (male $n=4$, female $n=4$ ) were tracked by GPS-tags with VHF transmitters (E-obs $\mathrm{GmbH}$; Germany) and manually restrained on a monthly basis for blood sampling. All eight animals were located on Picard Island of Aldabra Atoll $(-9.225925,46.125266)$ and were individually identified by a branded three-digit mark on their carapace. The animals were considered sexually mature based on their carapace exceeding $55 \mathrm{~cm}$ straight length and/or age estimation based on the width of the third dorsal scute (Bourn, 1977; Grubb, 1971). The intermittent encounters with the animals did not allow for continuous observations of reproductive behaviour throughout this time.

\section{Sampling}

Monthly blood sampling was performed under manual immobilisation in lateral position. Manual stabilisation and blood sampling from the proximal foreleg (vena brachialis) with $3 \mathrm{ml}$ syringes and hypodermic $19 \mathrm{G}$ and $23 \mathrm{G}$ needles did not exceed 15 minutes to avoid stressinduced changes in steroid hormone levels (Lance et al., 2004). Blood was immediately transferred into lithiumheparin containing tubes (Vacuette Tubes, Greiner BioOne Vacuette, Switzerland) and stored in a thermosbox with ice bricks for transport to the Aldabra Atoll laboratory. Plasma was centrifuged within $2 \mathrm{hrs}$ of collection and stored at $-20^{\circ} \mathrm{C}$ until further analysis.

\section{Enzyme-linked immunosorbent assay (ELISA) for steroid hormones}

In males, testosterone was measured and in females, oestradiol and progesterone were measured. To minimise repeated thawing of the samples, whenever possible multiple hormones were measured within each sample on the same day. Oestradiol concentrations were quantified using a commercially available EIA kit (Arbor Assays, Ann Arbor, Michigan, USA; KB30-H1) as per manufacturer's instructions. Testosterone and progesterone concentrations were quantified using EIA methods described previously (Kummrow et al., 2011). Antisera (C. Munro, University of California, Davis, California, USA) were diluted as follows: $T$ (polyclonal R156/7), 1:10,000 and P (monoclonal CL425, Quidel Corp., California, USA with final purification by C. Munro), $1: 8,800$. Horseradish peroxidase conjugates (C. Munro) were diluted as follows: T-HRP, 1:20,000 and P-HRP, 1:40,000. Standards used were T (Steraloids Inc., A6950; 48-12,500 pg/ml) and P (Sigma P0130; 15.6-4,000 pg/ $\mathrm{ml}$ ). Samples were run neat in EIA buffer ( $0.1 \mathrm{mM}$ sodium phosphate buffer, $\mathrm{pH} 7.0$, containing $9 \mathrm{~g}$ of $\mathrm{NaCl}$ and 1 $\mathrm{g}$ of BSA per litre). Controls consisted of laboratory stocks of pooled samples obtained from cycling females and males from a variety of species, and run at 30 and $70 \%$ binding. Standards, samples and controls were run in duplicate with $<10 \%$ CV between duplicates. Intraand inter-assay CVs were $<10 \%$ and $<15 \%$, respectively, for all assays.

The results were plotted graphically against previously reported behavioural and anatomical observations (Bourn, 1977; Swingland \& Coe, 1978).

\section{Meteorological data}

Measurements of daily temperature (mean, maximal, and minimal; $\left.{ }^{\circ} \mathrm{C}\right)$, precipitation $(\mathrm{mm} /$ day), and sunshine duration ( $\mathrm{min} /$ day) from the Aldabra Islands (LAT -9.72259, LON 47.0407) were acquired from a commercial data bank (Meteoblue AG, 4058 Basel, Switzerland), with mean values of 1985-2018 extracted and used for analysis. 


\section{RESULTS}

Female steroid hormone levels

Female plasma oestradiol levels are depicted in Figure 1 and varied between 130 and $310 \mathrm{pg} / \mathrm{ml}$. During the annual cycle, plasma oestradiol concentrations showed a seasonal pattern with high values in February and March, a decline in May and June, followed by the nadir in September and increasing values in November and December. Plasma progesterone levels were only measurable above detection limit $(19 \mathrm{pg} / \mathrm{ml})$ in April in one of the four animals $(480 \mathrm{pg} / \mathrm{ml})$.

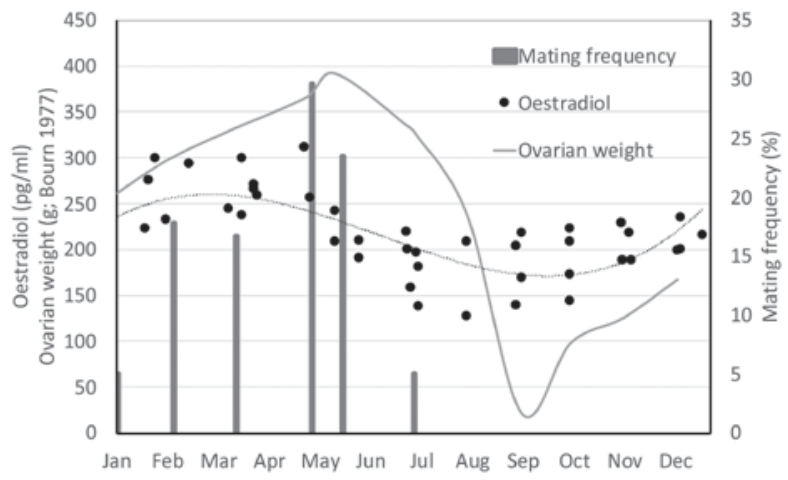

Figure 1. Plasma oestradiol (solid circles) with trendline (dotted line) of female Aldabra giant tortoises (A. gigantea, $\mathrm{n}=4$ ) over an entire year in relation to published ovarian weight and mating frequency, plotted as monthly fraction of the yearly mating occurrences (from Bourn, 1977).

\section{Male steroid hormone levels}

Plasma testosterone levels in male Aldabra giant tortoises are depicted in Figure 2 and ranged from 0.14 to $3.61 \mathrm{ng} /$ $\mathrm{ml}$, showing a seasonal variation with highest values in January and lowest values in September.

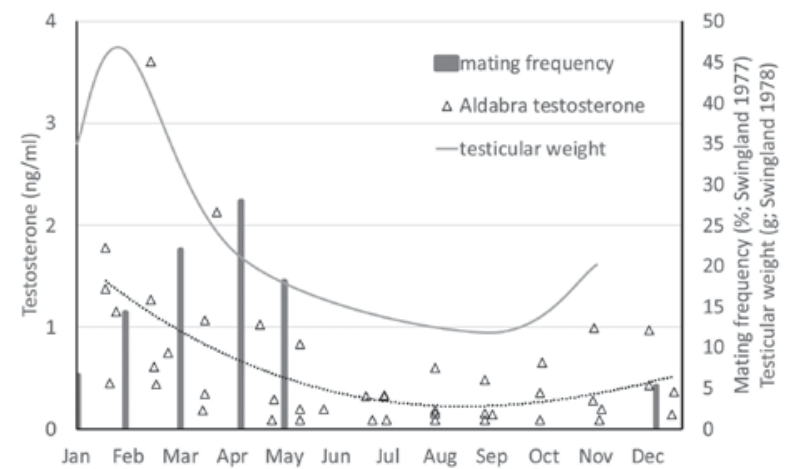

Figure 2. Plasma testosterone (triangles) with trendline (dotted line) of male Aldabra giant tortoises ( . gigantea, $\mathrm{n}=4$ ) over an entire year in relation to published mating frequency, plotted as monthly fraction of the yearly mating occurrences, and testicular weight (from Swingland and Coe, 1978).

\section{Meteorological data}

In April and May, the critical time for late folliculogenesis and ovulation, precipitation in the year 2013 was below the standard deviation of the mean precipitation of the years 1985 to 2018, and sunshine duration longer, whereas mean daily temperature 2013 was only slightly higher than the mean and remained within the standard deviation (Fig. 3).

A

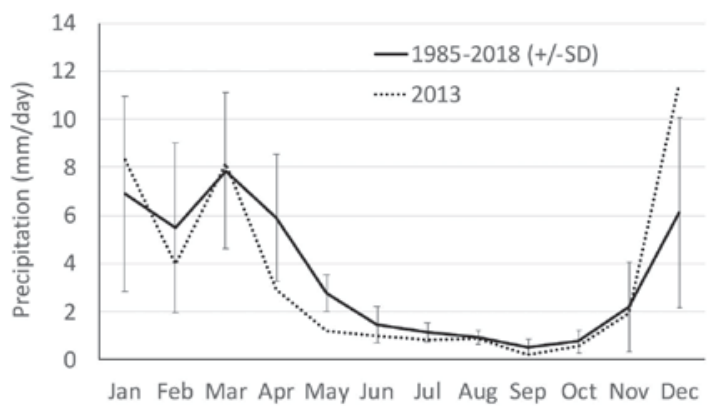

B

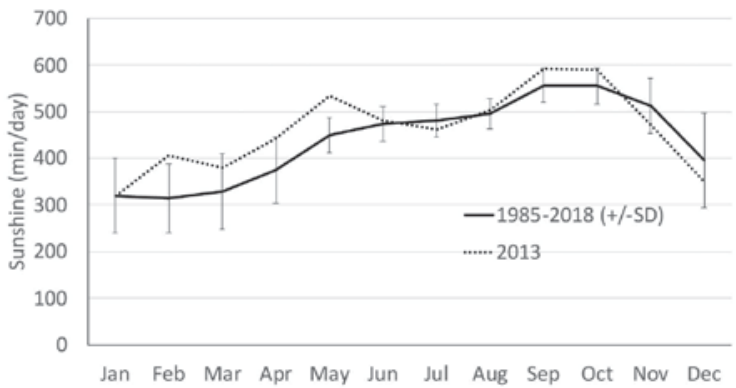

C

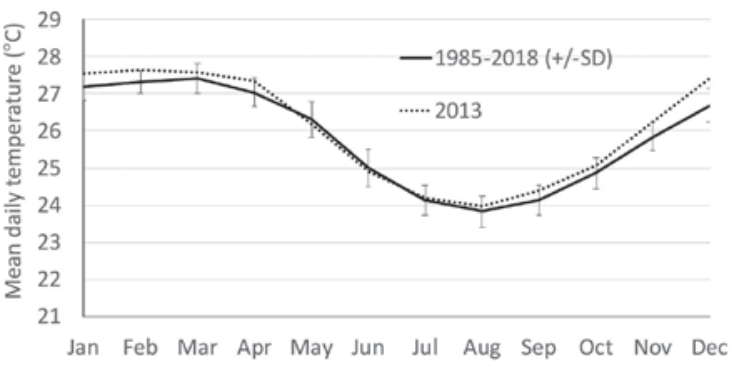

Figure 3. Meteorological data of the Aldabra Atoll, A) precipitation, B) daily sunshine duration, C) mean daily temperatures, mean values 1985-2018 (solid line, +/- SD) and the values of the year of sampling (2013, dotted line).

\section{DISCUSSION}

We report seasonal cyclicity in plasma oestradiol and testosterone in free-living Aldabra giant tortoise females and males, respectively, indicating a seasonal cycle of gonadal steroid hormones in their endemic range, coinciding with previously reported behavioural and anatomical observations (Bourn, 1977; Swingland \& Coe, 1978).

The Aldabra giant tortoises' island habitats are located within the southern hemisphere tropical climate zone, at geographic latitude $-9.23^{\circ}$. At such low latitudes, one would theoretically not expect a seasonal reproduction linked to a photoperiod signal, because the annual 
variation in daylength is not considered sufficient for such a signal below a latitude of $11.75^{\circ}$ (Bronson \& Heideman, 1994). Yet, Aldabra giant tortoises exhibit distinct seasonality in reproduction, seemingly regulated by or adapted to climatic patterns of temperature and rainfall and available food resources (Bourn, 1977; Haverkamp et al., 2017; Schramm et al., 1999; Swingland \& Coe, 1978). They show a peak in mating behaviour from February to May during the latter part of the rainy season, with the peak nesting season from June to September coinciding with the dry season, allowing the hatchlings to emerge at the beginning of the rainy season starting in October (Swingland \& Coe, 1978). While for cool-climate reptile species, female reproduction is naturally restricted to the warmest times of the year, both biotic (e.g. predation on eggs, hatchlings or nesting females) and abiotic factors (e.g. precipitation, temperature) have been discussed as hypotheses to explain seasonality in tropical reptiles species (Brown \& Shine, 2006).

Although not definitively investigated and proven, the timing of folliculogenesis and breeding activity during and at the end of the wet season was suggested to be associated with increased availability of food and other resources (Bourn, 1977). Seasonal changes in body condition of nesting females, and a positive association between body condition of nesting females and reproductive output, have been shown in several egglaying reptile species (Henen, 1997; Litzgus et al., 2008; Loehr et al., 2007).

Increasing and peak plasma oestradiol levels are indicative of follicular development/ vitellogenesis, and the decrease of oestradiol levels paired with a sharp increase in progesterone are indicative of ovulation in egg laying reptile species (Callard et al., 1991). The seasonal pattern of plasma oestradiol concentrations in all four Aldabra tortoise females concurred with the observed changes in ovarian weight (Bourn, 1977), with vitellogenesis during the winter months leading up to a peak in February to April. The observed peak in mating frequency, plotted as monthly fraction of the yearly mating occurrences (Bourn, 1977), coincided with the decrease in oestradiol, facilitating ovulation, and increasing oestradiol after the end of the oviposition phase in September (Bourn, 1977) indicated the initiation a new phase of follicular development.

Interestingly, although all four A. gigantea females demonstrated increase of plasma oestradiol indicative of follicular development, only one female showed a single increased progesterone value during the presumed phase of ovulation in May to June. Behavioural and morphological data from field studies suggested that food availability and, in their direct influence on resource availability, population density and rainfall are primary regulative factors in the reproductive output in Aldabra tortoises (Haverkamp et al., 2017; Swingland \& Coe, 1978). Although mature females, regardless of resource availability, showed the same reproductive potential, i.e. follicular development, those in high density populations regressed all or parts of their preovulatory follicles, whereas follicular atresia was not observed in the low-density populations (Swingland \& Coe, 1978).
In comparison to the mean values of the years 1985 to 2018, meteorological data of the year 2013 showed a distinctively lower precipitation and increased daily sunshine duration in April and May (Haverkamp et al., 2017), indicating an unusually dry season. Although only a small number of individuals were included in our study, our data may be indicative of a high rate of follicular atresia in 2013, presumably due to drought resulting in resource scarcity during the critical months in which ovulation would have occurred. Mean daily temperatures in 2013 did not differ from the mean values of 19852018, and are therefore less likely to qualify as a trigger factor for follicular atresia. Alternatively, methodological limitations, such as insufficient sensitivity of the ELISA to low levels of unbound circulating progesterone may have been responsible for the lack of progesterone data. Prior extraction of progesterone to expand measurement to protein-bound hormone metabolites may have increased the sensitivity of the ELISA and revealed seasonal peaks (Graham et al., 2016). The field conditions did not allow for storage at $-80^{\circ} \mathrm{C}$ and permanent freezing may not have always been guaranteed during transports, however, the impact of storage duration and temperature on blood steroid hormone levels have been reported to be minimal (Reyna et al., 2001; Taylor \& Schuett, 2004).

Plasma testosterone levels reflected spermiogenesis in the males with high values during the wet season, corresponding with the observed peak in testicular weight and mating frequency, and decreasing levels during the dry season when mating activity ceased (Swingland \& Coe, 1978).

The low number of animals and the study design based on monthly encounters with the animals for blood sampling without continuous monitoring for reproductive activity, resulting in a lack of biological validation of the hormonal data, are constraints of the study that limit the data interpretation to a descriptive study. Nevertheless, our study complements previous anatomic, behavioural and morphological studies with hormonal data and provides baseline data for endocrinological monitoring of reproductive processes and management in captive breeding programs.

\section{ACKNOWLEDGEMENTS}

We thank the Seychelles Islands Foundation for their collaboration and support of the project and their staff on Aldabra, especially Heather Richards, Marvin Roseline, and Sheril Decommarmond for their assistance and logistical support during our work on the Atoll, and Lindsay Turnbull for support of the project. Special thanks are extended to Christine Gilman for her assistance with the hormone analysis. Funding was provided by the Swiss National Science Foundation (grant number 31003A_143940), the 'Investitionskredit' of the University of Zurich, and the Zurich Zoo.

\section{REFERENCES}

Bourn, D. (1977). Reproductive study of giant tortoises on Aldabra. Journal of Zoology 182, 27-38. 
Bourn, D., Gibson, C., Augeri, D., Wilson, C. J., Church, J. \& Hay, S.I. (1999). The rise and fall of the Aldabran giant tortoise. Proceedings of the Royal Society of London 1091-1100.

Bronson, F. \& Heideman, P. (1994). Seasonal regulation of reproduction in mammals. Physiology of Reproduction 2, 541-584.

Brown, G. P. \& Shine, R. (2006). Why do most tropical animals reproduce seasonally? Testing hypotheses on an Australian snake. Ecology 87, 113-143.

Callard, I. P., Etheridge, K., Giannoukos, G., Lamb, T. \& Perez, L. (1991). The role of steroids in reproduction in female elasmobranchs and reptiles. Journal of Steroid Biochemistry and Molecular Biology 40, 571-575.

Casares, M. (1995). Untersuchungenzum Fortpflanzungsgeschehen bei Riesenschildkröten (Geochelone elephantopus und G. gianteus) und Landschildkröten (Testudo graeca und T. hermanni) anhand von Ultraschalldiagnostik und Steroidanalyse im Kot. Zoologischer Garten 65, 50-76.

Falcón, W., Baxter, R. H., Furrer, S., Bauert, M., Hatt, J.-M., Schaepman-Strub, G., Ozgul, A., Bunbury, N., Clauss, M. \& Hansen, D. M. (2018). Patterns of activity and body temperature of Aldabra giant tortoises in relation to environmental temperature. Ecology and Evolution 8, 2108-2121.

Falcón, W. \& Hansen, D. M. (2018). Island rewilding with giant tortoises in an era of climate change. Philosophical Transactions of the Royal Society B: Biological Sciences 373, 20170442.

Falcón, W., Hansen, D. M. \& Moll, D. (2018). Frugivory and seed dispersal by chelonians: A review and synthesis. Biological Reviews 95, 142-166.

Gerlach, J., Rocamora, G., Gane, J., Jolliffe, K. \& Vanherck, L. (2013). Giant tortoise distribution and abundance in the Seychelles Islands: past, present, and future. Chelonian Conservation and Biology 12, 70-83.

Graham, K. M., Mylniczenko, N. D., Burns, C. M., Bettinger, T. L. \& Wheaton, C. J. (2016). Examining factors that may influence accurate measurement of testosterone in sea turtles. Journal of Veterinary Diagnostic Investigation 28, 12-19.

Grubb, P. (1971). Growth, ecology and population structure of giant tortoises on Aldabra. Philosophical Transactions of the Royal Society of London 260, 327-372.

Hansen, D. M., Donlan, C. J., Griffiths, C. J. \& Campbell, K. J. (2010). Ecological history and latent conservation potential: large and giant tortoises as a model for taxon substitutions. Ecography 33, 272-284.

Haverkamp, P. J., Shekeine, J., de Jong, R., Schaepman, M., Turnbull, L. A., Baxter, R., Hansen, D., Bunbury, N., FleischerDogley, F. \& Schaepman-Strub, G. (2017). Giant tortoise habitats under increasing drought conditions on Aldabra Atoll-Ecological indicators to monitor rainfall anomalies and related vegetation activity. Ecological Indicators 80 , 354-362.
Henen, B. T. (1997). Seasonal and annual energy budgets of female desert tortoises (Gopherus agassizii). Ecology 78, 283-296

Kummrow, M. S., Gilman, C., Mackie, P., Smith, D. A. \& Mastromonaco, G. F. (2011). Noninvasive analysis of fecal reproductive hormone metabolites in female veiled chameleons (Chamaeleo calyptratus) by enzyme immunoassay. Zoo Biology 30, 95-115.

Lance, V. A., Elsey, R. M., Butterstein, G. \& Trosclair lii, P. L. (2004). Rapid suppression of testosterone secretion after capture in male American alligators (Alligator mississippiensis). General and Comparative Endocrinology 135, 217-222.

Litzgus, J. D., Bolton, F. \& Schulte-Hostedde, A. I. (2008). Reproductive output depends on body condition in spotted turtles (Clemmys guttata). Copeia 2008, 86-92.

Loehr, V., Hofmeyr, M. \& Henen, B. (2007). Annual variation in the body condition of a small, arid-zone tortoise, Homopus signatus signatus. Journal of Arid Environments 71, 337-349.

Reyna, R., Traynor, K. D., Hines, G., Boots, L. R. \& Azziz, R. (2001). Repeated freezing and thawing does not generally alter assay results for several commonly studied reproductive hormones. Fertility and Sterility 76, 823-825.

Rostal, D. C., Robeck, T. R., Grumbles, J. S., Burchfield, P. M. \& Owens, D. W. (1998). Seasonal reproductive cycle of the Galapagos tortoise (Geochelone nigra) in captivity. Zoo Biology 17, 505-517.

Schramm, B. G., Casares, M. \& Lance, V. A. (1999). Steroid levels and reproductive cycle of the Galapagos tortoise, Geochelone nigra, living under seminatural conditions on Santa Cruz Island (Galapagos). General and Comparative Endocrinology 114, 108-120.

Swingland, I. R. \& Coe, M. (1978). The natural regulation of giant tortoise populations on Aldabra atoll. Reproduction. Journal of Zoology 186, 285-309.

Taylor, E. N. \& Schuett, G. W. (2004). Effect of temperature and storage duration on the stability of steroid hormones in blood samples from western diamond-backed rattlesnakes (Crotalus atrox). Herpetological Review 35, 14.

Turnbull, L. A., Ozgul, A., Accouche, W., Baxter, R., ChongSeng, L., Currie, J. C., Doak, N., Hansen, D. M., Pistorius, P. \& Richards, H. (2015). Persistence of distinctive morphotypes in the native range of the CITES-listed Aldabra giant tortoise. Ecology and Evolution 5, 5499-5508.

Walton, R., Baxter, R., Bunbury, N., Hansen, D., Fleischer-Dogley, F., Greenwood, S. \& Schaepman-Strub, G. (2019). In the land of giants: habitat use and selection of the Aldabra giant tortoise on Aldabra Atoll. Biodiversity and Conservation 28, 3183-3198.

ZIMS, Species360 Zoological Information Management System (2018). https://zims.species360.org.

Accepted: 30 May 2020 